Original Article

\title{
Identifying preventative measures against frailty, locomotive syndrome, and sarcopenia in young adults: a pilot study
}

\author{
Tomohiro YASUdA, $\mathrm{PhD}^{1)}$ \\ 1) School of Nursing, Seirei Christopher University: 3453 Mikatahara, Kita-ku, Hamamatsu, Shizuoka \\ 433-8558, Japan
}

\begin{abstract}
Purpose] The purpose of this study was to propose potential preventive measures against future mobility impairments and muscle loss in healthy young adults by comprehensively evaluating their status of frailty, locomotive syndrome, and sarcopenia. [Participants and Methods] A total of 83 Japanese young adults were enrolled in this study and evaluated using the diagnostic criteria for frailty (Japanese version of the Cardiovascular Health Study), locomotive syndrome (calculated as scores of the LOCOMO-25, the stand-up test, and two-step test), and sarcopenia (handgrip strength, usual gait test, skeletal muscle index). [Results] The prevalence of frailty-prefrailty (45.9\%) was higher than that of presarcopenia (22.3\%) and locomotive syndrome (14.1\%). The prevalence of combinations of frailty-prefrailty and presarcopenia; frailty-prefrailty and locomotive syndrome; and frailty-prefrailty, locomotive syndrome, and pre-sarcopenia was $9.4 \%, 4.7 \%$, and $3.5 \%$, respectively. [Conclusion] Only $40 \%$ of the participants did not meet the three diagnostic criteria. On the other hand, there were not many participants with sarcopenia and/or locomotive syndrome diagnoses, suggesting that the improvement in intrinsic skeletal muscle mass rather than physical function is important for healthy Japanese young adults and could possibly be protective against future mobility or muscle-loss disorders.

Key words: Frailty, Locomotive syndrome, Sarcopenia
\end{abstract}

(This article was submitted Jun. 13, 2021, and was accepted Aug. 2, 2021)

\section{INTRODUCTION}

Loss of skeletal muscle mass and sarcopenia are related to adverse health effects including falls, fractures, disability, and heart disease ${ }^{1-3)}$, as well as increased healthcare costs and decreased healthy life expectancy ${ }^{4}$. Therefore, determining preventive measures against sarcopenia is particularly important in avoiding future mobility and frailty problems in currently healthy young adults. Thus, the European Working Group on Sarcopenia in Older People (EWGSOP) and the Asian Working Group for Sarcopenia (AWGS) introduced recommendations for evaluating skeletal muscle index (SMI, defined by appendicular skeletal muscle mass/height ${ }^{2}$ ), muscle strength (handgrip), and physical capability (gait speed) for diagnosing and assessing sarcopenia ${ }^{5,6}$. Recent studies revealed that the ratio of presarcopenia (low SMI without impact on muscle strength or physical capability) was found in approximately one to two-thirds of healthy young females in Japan ${ }^{7,8)}$.

Frailty is known to be highly prevalent in older adults and is a risk factor adverse health outcomes, including falls, hospitalization, and mortality ${ }^{9}$. Therefore, frailty should be identified as early as possible, and preventive measures using effective criteria are needed to support healthcare in aging populations ${ }^{10,11)}$. The Japanese Orthopedic Association (JOA) proposed the concept of locomotive syndrome (LS) in $2007^{12}$ ), defined as reduced mobility due to impaired locomotor organs. Preventive measures against LS should become a meaningful precedent not only for Japan, but also for other countries with rapidly aging societies. Therefore, preventive actions against frailty and LS should be taken from a young age, in order to avoid future mobility issues or diagnoses like sarcopenia. 
Since frailty, LS, and sarcopenia do not exist independently of each other, preventative measures should protect against all three dysfunctions, despite the fact that their exact relationships to each other are unclear. Thus, the purpose of this study was to propose potential preventive measures against future mobility impairments and muscle loss in healthy young adults by comprehensively evaluating their status of frailty, locomotive syndrome, and sarcopenia. In this study, we decided that the three diagnostic criteria mainly used in older adults are also applied to young adults, because it has been reported that there are a certain proportion of even young adults who have presarcopenia ${ }^{7,8)}$ or locomotive syndrome ${ }^{13)}$. This suggests that it is important to examine the values of the three diagnostic criteria regardless of age. The originality and novelty of this study is that 1) it was targeted at young adults and 2) it comprehensively utilized of the three diagnostic criteria to understand the current state of bedridden prevention from an early stage of lifestyle.

\section{PARTICIPANTS AND METHODS}

A total of 83 Japanese male and female university students aged 18-20 years were recruited through oral communications in a "sports practice" course at our university. There were no participants with musculoskeletal disease and a history of knee joint surgery, all participants took "sports practice" without any problems. Before informed consent was obtained, a written description of the purpose of the study and its safety was distributed to potential participants, along with a lifestyle questionnaire. The participants in this study were classified as "recreationally active"; 46 (12 for male and 34 for female) of 83 participated in regular aerobic-type exercise (walking, jogging, or cycling; 2-3 times per week for approximately 30 minutes). None of the participants had participated in strength/resistance-type training for 6 months before the start of the study. All participants were free of overt chronic disease (e.g., diabetes, angina, myocardial infarction, cancer, and stroke) as assessed by their annual medical examination. All 83 young adults included who met the criteria were included in the data analyses. The principles of the World Medical Association Declaration of Helsinki and the American College of Sports Medicine Guidelines for the Use of Human Participants were adopted in this study. The study was approved by the ethics committee of the Seirei Christopher University (approval number: 19087).

The Japanese version of the Cardiovascular Health Study (J-CHS) criteria questionnaire were as follows, with the respective answers that indicated potential frailty: (1) Have you lost $2 \mathrm{~kg}$ or more in the past 6 months? "Yes", (2) Do you engage in moderate levels of physical exercise or sports aimed at health? Do you engage in low levels of physical exercise aimed at health? "No" to both questions, (3) In the past 2 weeks, have you felt tired without a reason? "Yes", (4) grip strength $<26 \mathrm{~kg}$ in men or $18 \mathrm{~kg}$ in women, and (5) gait speed $<1.0 \mathrm{~m} / \mathrm{s}$. Frailty, prefrailty, and robustness were defined by having three to five, one to two, and zero items, respectively ${ }^{11)}$.

The diagnostic criteria for LS were as follows: (1) self-rated locomotive function, (2) stand-up ability (knee extension torque), and (3) walking ability. These outcomes were calculated as scores of the LOCOMO-25, stand-up test, and two-step test, respectively. The scoring criteria of LOCOMO-25 followed the 25 -question geriatric locomotive function scale ${ }^{14)}$. The scoring criteria of the stand-up test and two-step test followed the scoring system of each test ${ }^{15}$. Based on the scores of three tests as a guide to assessing the risk of locomotive syndrome, the scores was classified into three stages as follows. Stage 1, if any of the three conditions (a-c) were met: (a) stand-up test, difficulty in standing from a 40-cm high seat using one-leg (either leg); (b) two-step test, $<1.3$; (c) 25-question score, $\geq 7$ ). Stage 2, if any of the three conditions (a-c) were met: (a) stand-up test, difficulty in standing from a 20-cm high seat using both legs (either leg); (b) two-step test, <1.1; (c) 25-question score, $\geq 16$ ). Robust: did not meet criteria for stages 1 or 2 . The diagnostic criteria were reported previously ${ }^{16)}$.

Body mass and standing height were measured to the nearest $0.1 \mathrm{~kg}$ and $0.1 \mathrm{~cm}$, respectively, by using a standing height scale and an electronic weight scale. Body mass index (BMI) was defined as body mass $/ \mathrm{height}^{2}\left(\mathrm{~kg} / \mathrm{m}^{2}\right)$. A multi-frequency bioelectrical impedance analyzer (BIA), InBody 430 analyzer (Biospace Co., Ltd., Seoul, Korea) was used according to the manufacturer's guidelines. BIA is used to estimate body composition according to the difference in conductivity of the various tissues based on the differences in their biological characteristics. This body composition analyzer adopts a tetrapolar, eight-point tactile electrode system that separately measures impedance of the arms, trunk, and legs at three different frequencies $(5,50$, and $250 \mathrm{kHz})$ for each segment. The measurements were carried out while the participants rested quietly in the supine position, with their elbows extended and relaxed along their trunk. The InBody automatically estimates body weight, BMI, percent body fat, and lean soft tissue of the two upper limbs and two lower limbs. The skeletal muscle index (SMI; $\mathrm{AMM} / \mathrm{height}^{2}, \mathrm{~kg} / \mathrm{m}^{2}$ ) was calculated as the sum of the two upper limbs and two lower limbs (AMM) ${ }^{5-7)}$. The usual gait test was measured over a 6-m course ${ }^{5,6}$.

Handgrip strength was measured using a factory-calibrated hand dynamometer (TKK 5401; Takei, Tokyo, Japan). All participants were instructed to maintain an upright standing position, arms at their sides, holding the dynamometer in the right hand with the elbow extended at $180^{\circ}$ without squeezing their arm against their body. The size of the dynamometer's handle was set to feel comfortable for the participant while squeezing the grip. Each participant underwent two trials, and the best value of each trial was used for analysis ${ }^{7}$.

Results are expressed as mean \pm standard deviation for all variables. All data were analyzed using JMP software (version 12.0 SAS Institute Inc., Tokyo, Japan). When the data were not normally distributed, non-parametric statistical analysis (Wilcoxon signed rank test) was used to identify differences between male and female groups. Statistical significance was set at $p<0.05$. Differences in each diagnostic criterion between males and females were examined using the $\chi^{2}$ test. 


\section{RESULTS}

Physical characteristics and clinical data are presented in Table 1. Anthropometry and morphological assessments were significantly higher in men than in women, except for BMI, thigh girth, and anterior thigh MTH (Table 1). There were no differences in functional assessments between males and females, except for handgrip and two-step test scores (Table 1). In J-CHS criteria questionnaire, there were no participants applying to "(5) gait speed $<1.0 \mathrm{~m} / \mathrm{s}$ ". In locomotive syndrome criteria, there were no participants applying to "(a) stand-up test, difficulty in standing from a 40-cm high seat using one-leg (either leg)" and "(b) two-step test, $<1.3$ ". There were no significant differences in the ratios of frailty ( $\mathrm{p}=0.076)$, locomotive syndrome $(\mathrm{p}=0.274)$, and sarcopenia $(\mathrm{p}=0.478)$ between the male and female groups (Tables $2-4)$. The prevalence of frailty-prefrailty (45.9\%) was higher than that of presarcopenia $(22.3 \%)$ and locomotive syndrome (14.1\%). The prevalence of combining frailty-prefrailty and pre-sarcopenia, combining frailty-prefrailty and locomotive syndrome, or combining frailty-prefrailty, locomotive syndrome, and presarcopenia was $9.4 \%, 4.7 \%$, and 3.5\%, respectively (Fig. 1).

\section{DISCUSSION}

The main findings of this study are as follows: First, approximately $60 \%$ of healthy Japanese young adults were identified as having any or all three diagnostic criteria. Second, there were not many participants with stage 2 sarcopenia and/or locomotive syndrome, unlike prefrailty and presarcopenia.

Table 1. The physical characteristics and clinical data in Japanese university male and female

\begin{tabular}{lccc}
\hline Variable & Male & Female & p-value \\
\hline $\mathrm{N}$ & 18 & 65 & - \\
Age, years & $18.7(1.2)$ & $18.4(0.6)$ & 0.526 \\
Standing height, cm & $168.9(6.7)^{* *}$ & $156.9(5.0)$ & 0.001 \\
Body weight, kg & $58.9(6.3)^{* *}$ & $51.5(7.8)$ & 0.001 \\
BMI, kg/m & $20.4(2.2)$ & $20.9(2.8)$ & 0.561 \\
\% body fat & $13.2(4.5)^{* *}$ & $26.3(6.0)$ & 0.001 \\
Fat mass, kg & $7.8(3.3)^{* *}$ & $13.9(5.3)$ & 0.001 \\
Morphological assessment & & & \\
Fat-free mass, kg & $28.1(3.1)^{* *}$ & $20.3(2.2)$ & 0.001 \\
SMI, kg/m & $7.47(0.57)^{* *}$ & $6.07(0.54)$ & 0.001 \\
Thigh girth, cm & $48.7(4.1)$ & $48.5(4.5)$ & 0.468 \\
Calf girth, cm & $35.4(2.0)^{*}$ & $34.2(2.9)$ & 0.043 \\
Anterior thigh MTH, cm & $4.80(0.65)$ & $4.50(0.64)$ & 0.099 \\
Posterior thigh MTH, cm & $6.08(0.50)^{* *}$ & $5.54(0.62)$ & 0.001 \\
Posterior calf MTH, cm & $6.58(0.45)^{* *}$ & $5.98(0.67)$ & 0.001 \\
Functional assessment & & & \\
J-CHS, unit/5-question & $0.7(0.8)$ & $0.7(0.8)$ & 0.613 \\
Handgrip, kg & $42.1(6.5)^{* *}$ & $27.0(5.9)$ & 0.001 \\
Gait speed, sec/5 m & $3.70(0.55)$ & $3.60(0.56)$ & 0.711 \\
Two-Step test, m/m & $1.71(0.14)^{*}$ & $1.64(0.10)$ & 0.014 \\
Stand-Up test, cm & $12.9(7.7)$ & $17.6(11.9)$ & 0.190 \\
25-Question GLFS, unit & $2.7(2.4)$ & $3.6(3.7)$ & 0.456 \\
\hline
\end{tabular}

Data are given as mean (standard deviation). BMI: Body mass index; J-CHS: Japanese version of the Cardiovascular Health Study; MTH: muscle thickness; SMI: skeletal muscle mass index. ${ }^{* *} \mathrm{p}<0.01,{ }^{*} \mathrm{p}<0.05$, male vs. female.

Table 2. Comparison based on diagnostic criteria and their proportions of frailty (J-CHS) in Japanese university male and female

\begin{tabular}{|c|c|c|c|c|c|c|}
\hline \multirow{2}{*}{$\begin{array}{l}\text { Categories } \\
\text { Number of items }\end{array}$} & \multirow{2}{*}{$\begin{array}{c}\text { Robust } \\
\text { None }\end{array}$} & \multicolumn{2}{|c|}{ Prefrailty } & \multicolumn{3}{|c|}{ Frailty } \\
\hline & & 1 & 2 & 3 & 4 & 5 \\
\hline Male $(\mathrm{n}=18)$ & $11(61.1 \%)$ & $4(22.2 \%)$ & $1(5.6 \%)$ & $2(11.1 \%)$ & $0(0.0 \%)$ & $0(0.0 \%)$ \\
\hline Female $(n=65)$ & $33(50.8 \%)$ & $21(32.3 \%)$ & $10(15.4 \%)$ & $1(1.5 \%)$ & $0(0.0 \%)$ & $0(0.0 \%)$ \\
\hline
\end{tabular}

J-CHS: Japanese version of the Cardiovascular Health Study. 
Table 3. Comparison based on diagnostic criteria and their proportions of locomotive syndrome in Japanese university male and female

\begin{tabular}{lccc}
\hline Category & Robust & Stage 1 & Stage 2 \\
\hline Male $(\mathrm{n}=18)$ & $18(100 \%)$ & $0(0.0 \%)$ & $0(0.0 \%)$ \\
Female $(\mathrm{n}=65)$ & $53(81.5 \%)$ & $11(16.9 \%)$ & $1(1.5 \%)$ \\
\hline
\end{tabular}

Table 4. Comparison based on diagnostic criteria and their proportions of sarcopenia in Japanese university male and female

\begin{tabular}{lccc}
\hline Category & Robust & Presarcopenia & Sarcopenia \\
\hline Male $(\mathrm{n}=18)$ & $15(83.3 \%)$ & $3(16.7 \%)$ & $0(0.0 \%)$ \\
Female $(\mathrm{n}=65)$ & $49(75.4 \%)$ & $16(24.6 \%)$ & $0(0.0 \%)$ \\
\hline
\end{tabular}

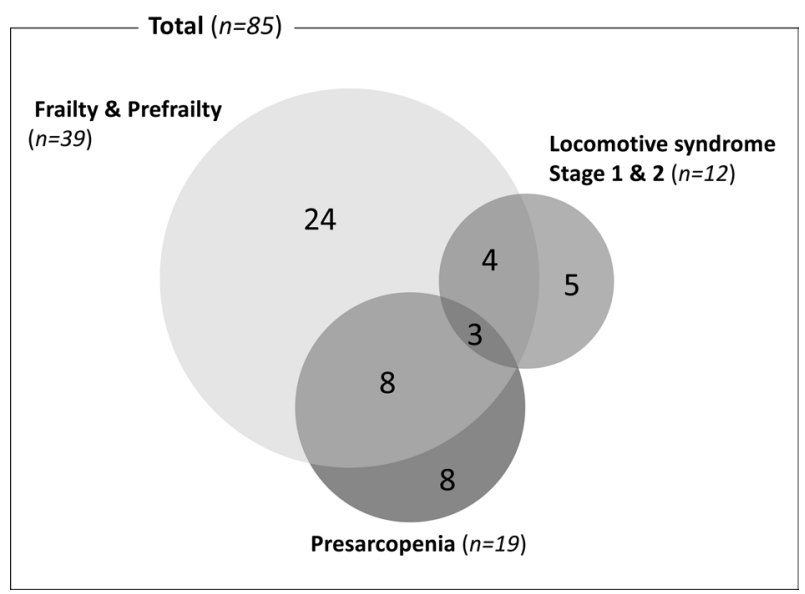

Fig. 1. The number of frailty-prefrailty, locomotive syndrome stage $1 \& 2$, and presarcopenia in healthy Japanese young adults $(n=85)$.

Standing height, body weight, and handgrip strength in the present study (Male: $168.9 \mathrm{~cm}, 58.9 \mathrm{~kg}, 20.4 \mathrm{~kg} / \mathrm{m}^{2}, 42.1 \mathrm{~kg}$; Female: $156.9 \mathrm{~cm}, 51.5 \mathrm{~kg}, 20.4 \mathrm{~kg} / \mathrm{m}^{2}, 27.0 \mathrm{~kg}$ ) were similar to the standard values for Japanese physical fitness (Male: $171.1 \mathrm{~cm}, 63.0 \mathrm{~kg}, 21.4 \mathrm{~kg} / \mathrm{m}^{2}, 45.8 \mathrm{~kg}$ [19 years]; Female: $158.7 \mathrm{~cm}, 52.2 \mathrm{~kg}, 20.7 \mathrm{~kg} / \mathrm{m}^{2}, 28.4 \mathrm{~kg}$ [19 years]) ${ }^{17)}$. Thus, it appears that the physical characteristics and muscle strength observed in this study represent the current general population of healthy Japanese young adults.

Interestingly, the prevalence of frailty and prefrailty was $46 \%$ in the young adults in this study. The concept of frailty includes "weight loss", "low levels of physical exercise" and "feeling tired", so there is a high possibility that even healthy young adults with no pre-disease states are vulnerable to these situations. J-CHS is one of the gold standards in Japan ${ }^{11)}$, but it is necessary to note that J-CHS contains multiple indices that could be applicable to young adults with no dysfunction.

As in previous studies ${ }^{7,8)}$, young adults were vulnerable to pre-sarcopenia but not to sarcopenia. In addition, there were few young adults who appeared to demonstrate stage 2 locomotive syndrome. In Japan, previous studies demonstrated a decrease in physical strength from a young age compared to over 30 years ago ${ }^{18)}$, but our results revealed that the effect of morphology (skeletal muscle mass) is greater than that of functional (muscle strength, activities of daily living, etc.). In general, regular medical examinations and physical fitness tests are thought to be one of the most important health markers regardless of age and gender, and they are widely performed using anthropometric measurements and field-based simplified methods at the national level in Japan ${ }^{19}$. However, only body weight measurement, not body fat percentage or skeletal muscle mass, is generally used in regular medical examinations in Japan ${ }^{20)}$. Evaluations using body fat percentage and skeletal muscle mass rather than body weight should be adopted for assessing health from a young age, as differentiating between a patient's lean muscle relative to fatty tissue could be a way to preventatively measures presarcopenia situations. For healthy young women, calf girth measurement is simplified morphological methods, but which could be an effective method to evaluate skeletal muscle mass even if the body fat percentage cannot be measured without a body composition analyzer ${ }^{21)}$. Taken together, these results suggest that the improvement of intrinsic skeletal muscle mass (resistance training, etc.) rather than physical function is important for healthy Japanese young adults to extend healthy life expectancy in the future.

The present study has some limitations. First, as the participants were young Japanese adults, the age distribution and 
physical characteristics were very limited. Second, the sample size was small. Future studies using a robust experimental design with a large sample size should be conducted to verify the findings of this study. In particular, the sample size of male participants was small, so it was difficult to discuss gender differences in this study. Therefore, additional research on these issues is required.

In conclusion, only approximately $40 \%$ of healthy Japanese young adults did not meet diagnostic criteria for frailty, sarcopenia, or LS. On the other hand, there were not many participants with existing sarcopenia and/or locomotive syndrome, suggesting that the improvement of intrinsic skeletal muscle mass rather than physical function is important for healthy Japanese young adults.

\section{Conference presentation}

A part of this study was presented at the 7th Annual Meeting of Japanese Association on Sarcopenia and Frailty 1st-15th December 2020, Tokyo, Japan.

\section{Funding}

This study was partially supported by Grant-in-aid (No. 18K10906) from the Japan Ministry of Education, Culture, Sports, Science, and Technology and by Seirei Christopher University Grants-in-Aid of Research.

\section{Conflicts of interest}

The author declares that he has no conflict of interest.

\section{REFERENCES}

1) Haykowsky MJ, Brubaker PH, Morgan TM, et al.: Impaired aerobic capacity and physical functional performance in older heart failure patients with preserved ejection fraction: role of lean body mass. J Gerontol A Biol Sci Med Sci, 2013, 68: 968-975. [Medline] [CrossRef]

2) Visser M, Goodpaster BH, Kritchevsky SB, et al.: Muscle mass, muscle strength, and muscle fat infiltration as predictors of incident mobility limitations in well-functioning older persons. J Gerontol A Biol Sci Med Sci, 2005, 60: 324-333. [Medline] [CrossRef]

3) Yasuda T, Nakajima T, Sawaguchi T, et al.: Short Physical Performance Battery for cardiovascular disease inpatients: implications for critical factors and sarcopenia. Sci Rep, 2017, 7: 17425. [Medline] [CrossRef]

4) Janssen I, Shepard DS, Katzmarzyk PT, et al.: The healthcare costs of sarcopenia in the United States. J Am Geriatr Soc, 2004, 52: 80-85. [Medline] [CrossRef]

5) Cruz-Jentoft AJ, Baeyens JP, Bauer JM, et al. European Working Group on Sarcopenia in Older People: Sarcopenia: European consensus on definition and diagnosis: report of the European Working Group on sarcopenia in older people. Age Ageing, 2010, 39: 412-423. [Medline] [CrossRef]

6) Chen LK, Liu LK, Woo J, et al.: Sarcopenia in Asia: consensus report of the Asian Working Group for Sarcopenia. J Am Med Dir Assoc, 2014, 15: 95-101. [Medline] [CrossRef]

7) Yasuda T: Anthropometric, body composition, and somatotype characteristics of Japanese young women: implications for normal-weight obesity syndrome and sarcopenia diagnosis criteria. Interv Med Appl Sci, 2019, 11: 117-121. [Medline]

8) Ayabe M, Kumahara H, Yamaguchi-Watanabe A, et al.: Appendicular muscle mass and exercise/sports participation history in young Japanese women. Ann Hum Biol, 2019, 46: 335-339. [Medline] [CrossRef]

9) Fried LP, Tangen CM, Walston J, et al. Cardiovascular Health Study Collaborative Research Group: Frailty in older adults: evidence for a phenotype. J Gerontol A Biol Sci Med Sci, 2001, 56: M146-M156. [Medline] [CrossRef]

10) Basic D, Shanley C: Frailty in an older inpatient population: using the clinical frailty scale to predict patient outcomes. J Aging Health, 2015 , 27 : $670-685$. [Medline] [CrossRef]

11) Satake S, Shimada H, Yamada M, et al.: Prevalence of frailty among community-dwellers and outpatients in Japan as defined by the Japanese version of the cardiovascular health study criteria. Geriatr Gerontol Int, 2017, 17: 2629-2634. [Medline] [CrossRef]

12) Nakamura K: A "super-aged" society and the "locomotive syndrome". J Orthop Sci, 2008, 13: 1-2. [Medline] [CrossRef]

13) Nishimura A, Ohtsuki M, Kato T, et al.: Locomotive syndrome testing in young and middle adulthood. Mod Rheumatol, 2020, 30: 178-183. [Medline] [CrossRef]

14) Siu PP, Cheung PW, Cheung JP: Validation of the LOCOMO-25 and its minimum clinically important differences in domain scores for Chinese patients with low back pain and neck pain. J Orthop Sci, 2019, 24: 1110-1117. [Medline] [CrossRef]

15) Nakamura K, Ogata T: Locomotive syndrome: definition and management. Clin Rev Bone Miner Metab, 2016, 14: 56-67. [Medline] [CrossRef]

16) Yoshimura $Y$, Ishijima M, Ishibashi M, et al.: A nationwide observational study of locomotive syndrome in Japan using the ResearchKit: the locomonitor study. J Orthop Sci, 2019, 24: 1094-1104. [Medline] [CrossRef]

17) Tokyo Metropolitan University: New Japanese physical fitness standard value II. Tokyo: Fumaidou Publishing. 2007, pp 21-165.

18) Ministry of Education, Culture, Sports, Science and Technology (MEXT): https://www.mext.go.jp/prev_sports/comp/b_menu/other/_icsFiles/afieldfile/2019/10/15/1421921_2.pdf(Accessed Jun. 8, 2021)

19) Ministry of Education, Culture, Sports, Science and Technology (MEXT): New Physical Fitness Test (1999). http://www.mext.go.jp/a_menu/sports/stamina/03040901.htm (Accessed Jun. 8, 2021)

20) Ministry of Education, Culture, Sports, Science and Technology (MEXT). https://www.mhlw.go.jp/file/06-Seisakujouhou-11200000-Roudoukijunkyoku/0000194701.pdf (Accessed Jun. 8, 2021)

21) Yasuda T: Simplified morphological evaluation of skeletal muscle mass and maximum muscle strength in healthy young women: comparison between thigh and calf. Wom Health Lond, 2020, 16: 1745506520962009. [Medline] 